\title{
A LITERATURE REVIEW OF ORGANISATIONAL, INDIVIDUAL AND TEAMWORK FACTORS CONTRIBUTING TO THE ICU DISCHARGE PROCESS
}

\author{
Francis Lin RN BMN MN(Hons) PhD Canditate \\ Wendy Chaboyer RN PhD \\ Marianne Wallis RN PhD
}

\section{INTRODUCTION}

Improving patient safety and patient outcomes has emerged as a priority for hospitals in the last 20 years. The US Institute of Medicine’s (IOM) report to Congress “To err is human” provided a coherent set of directions that set the agenda for patient safety worldwide. ${ }^{[1]}$ The IOM defines healthcare quality as "the degree to which health services for individuals and populations increase the likelihood of desired health outcomes and are consistent with current professional knowledge”. ${ }^{[2]}$ The IOM definition suggests a broad approach to measuring healthcare quality in terms of data-desired outcomes and related processes of care. The IOM's six aims of health care, safe, effective, patient-centred, timely, efficient, and equitable, ${ }^{[2]}$ provides an direction for improving patient safety and the quality of health care. It is against this context that a literature review of the ICU discharge process was conducted.

The Intensive Care Unit (ICU) is an essential component of most large hospitals in the modern healthcare system, providing critically ill patients with high quality care. In addition, patients undergoing major surgery often require ICU admission postoperatively. In Australia in 20042005, elective surgery accounted for $31.4 \%$ of ICU admissions and emergency cases accounted for $46.3 \%$ of ICU admissions. ${ }^{[3]}$ 
Intensive care resources are limited and expensive commodities. In 2002-2003, one study found that the average cost of an ICU bed in Australia was A\$2670 per day and the total stay per patient was A\$9852. ${ }^{[4]}$ Australia has significantly fewer ICU resources than other western countries. ${ }^{[5]}$ In 2002, the available ICU beds per 100,000 population was 25 in Germany, 24 in USA, 11 in Switzerland, and 10 in The Netherlands. ${ }^{[5]}$ In contrast, Martin et al. ${ }^{[3]}$ reported that in 2004-2005, there were only 6.1 ICU beds per 100,000 populations in privately and publicly funded institutions in Australia. Using the number of beds per population to argue shortage of beds is debatable, because research shows that patient acuity is lower in countries with more ICU beds. This may indicate that some ICU resources might be more optimally utilised. ${ }^{[6]}$ However, a lack of beds relative to population and the high cost suggests that optimal use of the existing ICU beds is imperative in coping with the increasing demand for ICU beds in Australia.

It would appear that optimal bed flow is critical to ensure high quality of care under current ICU capacities, given that ICUs are often under forward pressure from areas such as Emergency Department (ED) or Operating Theatre (OT) for beds ${ }^{[7]}$. Discharging patients is one way to relieve this pressure but clearly the risk of premature discharg ${ }^{[8]}$ must be managed. At the same time, lack of beds in other parts of the hospital can also cause discharge delays. One study identified that $46 \%$ of unsuccessful discharges from ICU were due to a lack of ward beds or disagreement over admitting services in the wards, and one in six discharges were unsuccessful on the first attempt. ${ }^{[9]}$ Often patients cannot be admitted into ICU because it is full, which may be because the ICU beds have been taken by patients waiting for ward beds, a situation referred to as discharge delay, "bed-block" or outflow limitation. ${ }^{[10,11]}$ On occasion, a patient maybe discharged prematurely to the ward because a sicker patient from ED or OT needs the ICU bed.

Discharging an ICU patient is a complex, multidisciplinary process, involving collaboration among physicians, nurses, managers, ward clerks, and support systems, both in ICU and across other hospital departments. Effective teamwork and coordination among staff can optimise the 
ICU patient discharge process and patient outcomes. The following section discusses the conceptual framework for this literature review.

\section{The conceptual framework of the review: Factors influencing ICU patient discharge processes}

The ICU patient discharge process may begin with a patient's admission to ICU when some of the discharge paperwork is started, and does not finish until the patient is transferred to the ward. Many factors can potentially cause problems. In the last two decades there has been increasing interest in researching factors that may contribute to patient outcomes in hospitals. ${ }^{[12-15]}$ In the 1990s, Reason ${ }^{[13,16]}$ identified that adverse events in complex healthcare systems may result from either active or latent failures. Active failures in a hospital setting are usually "committed" by the person closest to the patient, and this can lead to immediate adverse patient events. Latent failures, in contrast, refer to less apparent failures of organisation or design that contributed to the occurrence of errors. ${ }^{[17]}$ Latent failures often arise from management decisions that determine working conditions. Although active failures are much easier to identity than latent failures, identifying the latter could have a much larger effect on improving the working environment and patient safety.

Following this work, Vincent ${ }^{[14]}$ and Pronovost et al. ${ }^{[15]}$ identified a framework of six factors that may contribute to adverse events in clinical practice. The factors included: (i) patient factors, including clinical conditions, language, and social factors; (ii) task factors, including availability or use of protocols, test results, and accuracy of test results; (iii) individual factors, including knowledge, skills, competence, fatigue, failure to follow established protocols/procedures, motivation and attitude, and physical, mental health; (iv) teamwork factors, including verbal or written communication during handover, routine care and crisis, supervision and seeking help, and team structure and leadership; (v) working conditions, including staffing levels, skills mix, 
workload, availability or maintenance of equipment, and administrative and managerial support; and (vi) organisational and management factors, including financial resources, time pressures, and physical environment.

Based on these earlier frameworks, in this literature review, factors contributing to the ICU patient discharge process were grouped into four broad domains: organisational factors, individual factors, teamwork factors and patient factors (See Figure 1). Working conditions, organisational and management factors together were considered as organisational factors. The aim of this literature review was to critically analyse current literature related to factors that influence the ICU patient discharge process. It examined how organisational factors, individual factors and teamwork factors influence the ICU patient discharge. Patient factors, a widely wellresearched topic, ${ }^{[18,19]}$ were excluded to limit the review to a manageable length.

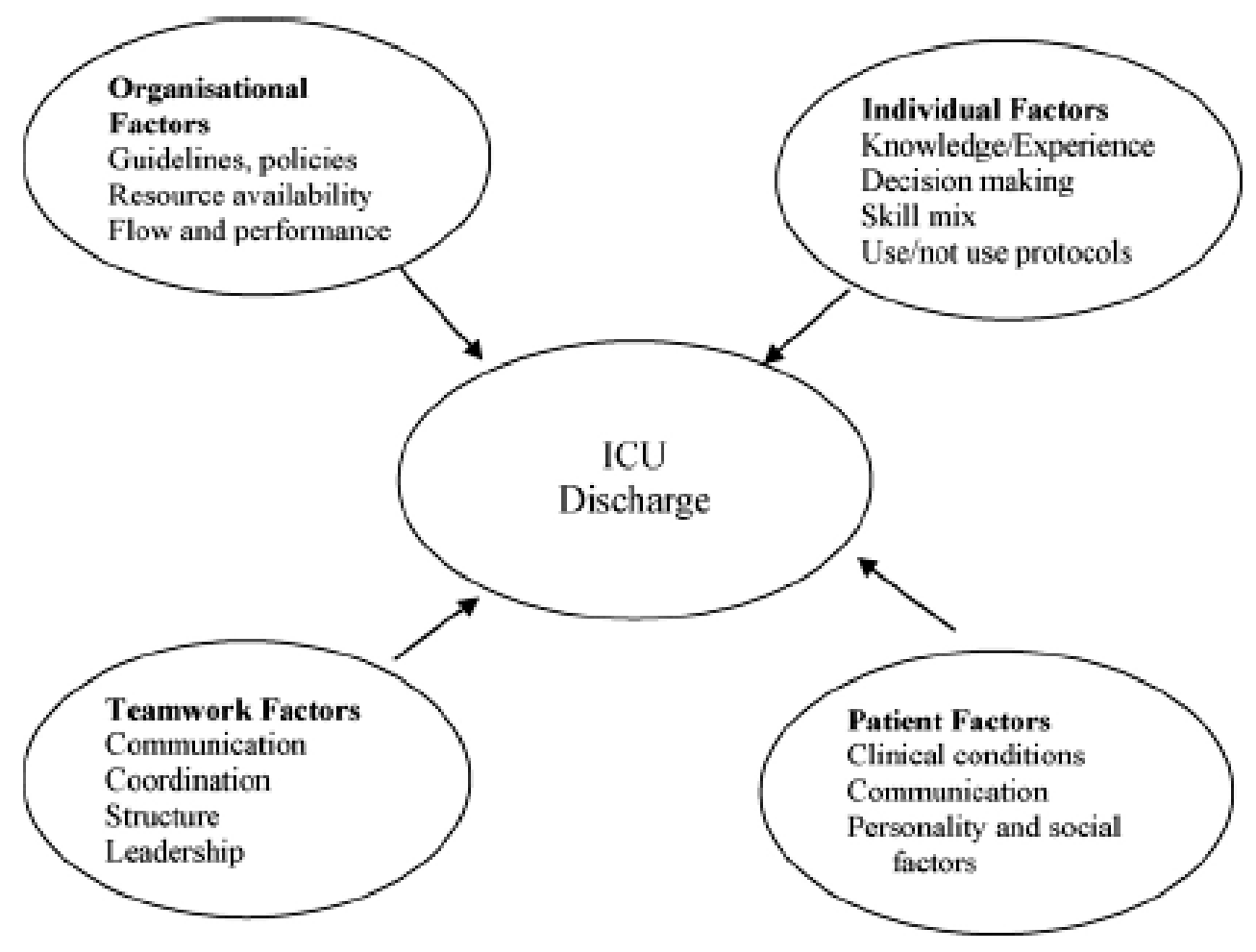

Figure 1 The ICU patient discharge process conceptual framework. 


\section{METHODS}

Databases including CINAHL, MEDLINE, PROQUEST, SCIENCE DIRECT were searched using key terms such as ICU discharge, discharge process, discharge policies, ICU guidelines and policies, discharge decision making, ICU and organisational factors, ICU and human factors, and ICU patient transfer. Web search and the "snow-balling" search of reference lists of articles were also used to locate relevant literature.

On the basis of this literature, the "ICU patient discharge process conceptual framework" was devised to facilitate the inclusion of articles for the review. Published policy, guidelines, and government reports related to ICU or High Dependency Unit (HDU) patient discharge, primary research articles on the influence of organisational factors, individual factors, and teamwork on ICU patient discharge processes and subsequent patient and organisational outcomes were included with no limitation on the year of publication. Articles were included if they were: 1) either qualitative or quantitative studies; 2) carried out in any type of ICU/HDU; and 3) directly related to the ICU patient discharge process. Articles were excluded if they were not specifically about ICU/HDU patient discharge. A total of 21 articles were included in the review.

\section{RESULTS}

Among the included articles, ten articles were on organisational factors including four reports, policies and guidelines, and organisational interventions regarding ICU discharge (see Table-1), five were on individual factors (see Table-2), and six were on teamwork factors (see Table-3).

\section{Organisational factors}

Articles on guidelines and policies, resource availability, and organisational interventions on flow and performance, were included under one broad domain as organisational factors (see Table-1) 
for discussion. Reports, policies and guidelines articles are not listed in the Table-1 due to the large amount of information included and the word limits of this paper.

\section{Guidelines and policies}

Only a few critical care organisations have written guidelines for the ICU patient discharge process. The Society of Critical Care Medicine (SCCM $)^{[20]}$ provides the most detailed admission and discharge criteria, focussing on diagnostic groups, clinical judgement of the need to support or monitor organ functioning, objective physiological parameters, and the stability or instability of physical conditions. According to the SCCM, ICU patients can be discharged when they are deemed to "no longer need ICU services". However, when there are limited ICU beds available, patients can be admitted and discharged by triage instead of through routine review processes. Due to the risks associated with triage, such as premature discharge ${ }^{[8]}$ the SCCM recommends that only ICU directors have the authority to make the decision to admit and discharge patients using this method.

The Intensive Care Society (ICS) ${ }^{[21]}$ Guidelines on Admission and Discharge to ICU and High Dependency Unit recommends the type of patient that should be admitted to the ICU; however, discharge criteria or guidelines are not specified. The Australian and New Zealand Intensive Care Society (ANZICS) has issued a document on minimum standards for ICUs which specifies that all ICUs should have clearly defined policies for discharge of patients, but it does not have a guideline for ICU patient discharge. ${ }^{[22]}$ Although guidelines and policies are considered effective management tools to reduce ICU length of stay and improve the utilisation of ICU resources, ${ }^{[23-}$ ${ }^{25]}$ one study indicated that only 18 out of 46 (39\%) ICUs had written discharge criteria. ${ }^{[26]}$ Heidegger et al. ${ }^{[27]}$ suggested that lack of agreement in clinical decision making exists in ICU patient discharge. 


\section{$\underline{\text { Resources }}$}

Many studies found that discharges from ICU at night were associated with increased mortality. ${ }^{[8,}$ ${ }^{28-31]}$ Goldfrad and Rowan ${ }^{[28]}$ identified that patients discharged from ICU at night experienced 2.5-fold greater mortality than patients discharged during the day. They also found that only $44.1 \%$ of patients discharged at night were fully ready to be discharged, compared with $86.3 \%$ of those discharged during the day. In an Australian study, Duke et al. ${ }^{[8]}$ found that patients discharged to the ward during the night shift had higher Acute Physiology and Chronic Health Evaluation (APACHE II) scores ${ }^{[32]}$ and crude mortality (no statistical significance). Their research results suggested that there was a higher prevalence of delayed discharge (37\%) and premature discharge (2\%) in association with night-shift discharge.

However, after-hours discharge still exists. In one Australian ICU, between 1992-2002, the number of patients being discharged to wards after-hours was $22 \%$ on evening shift (between 1500-2200 hours) and 6\% on night shift (between 2200-0800 hours). ${ }^{[31]}$ In 2003-2005, out of 70749 episodes of ICU care within a 12-month period in all Australian ICUs, 3036 (4.3\%) cases were discharged out-of-hours. ${ }^{[3]}$

At other times, patients were unable to be discharged because of resource constraints on the wards. Williams and Leslie ${ }^{[33]}$ found that $81 \%$ of delayed ICU discharges were due to a lack of available beds in the hospital and average delay time for ICU patient discharge was 21.3 hours. Levin $^{[9]}$ found that $16 \%$ of planned ICU discharges were unsuccessful on the first attempt due to lack of ward beds. It appears that ICU patient discharge and bed flow is largely influenced by the resource constraints of hospitals and the ICUs. After-hours discharge, although proved to be associated with increased mortality, coexists with the shortage of ICU beds. 


\section{Organisational interventions}

The articles on organisational interventions that influence ICU discharge are summarised in Table 1. Many researchers have studied interventions to improve discharge processes in order to improve patient safety and make more resources available for those patients who desperately need ICU service. ${ }^{[11,34,35]}$ Crocker and Keller ${ }^{[36]}$ reported that after removing the unnecessary steps in the patient discharge process, communication among staff improved and the patient journey was much smoother. Chaboyer et al. ${ }^{[11]}$ found that the use of an ICU liaison nurse could significantly reduce the discharge delay and improve ICU nurses perceptions of discharge planning; the liaison nurse coordinated the patient transition from ICU to ward and ensured continuity of patient care. Perlmutter et al. ${ }^{[35]}$ trialled a programme to identify the causes of discharge delays in one American neonatal ICU. Identification of problems and implementation of strategies to improve the patient discharge process reduced discharge delays and resulted in cost savings of $\$ 184,745$ for one year for the hospital. The use of an ICU outreach team, trialled by various researchers in the $U K,{ }^{[34,37,38]}$ was shown to decrease the patient hospital mortality rate. Ball et al. ${ }^{[34]}$ found that the ICU outreach team, which reviewed patients on the wards after discharge from ICU, increased the patients' hospital survival by 6.8\%, although this trend did not reach statistical significance. 


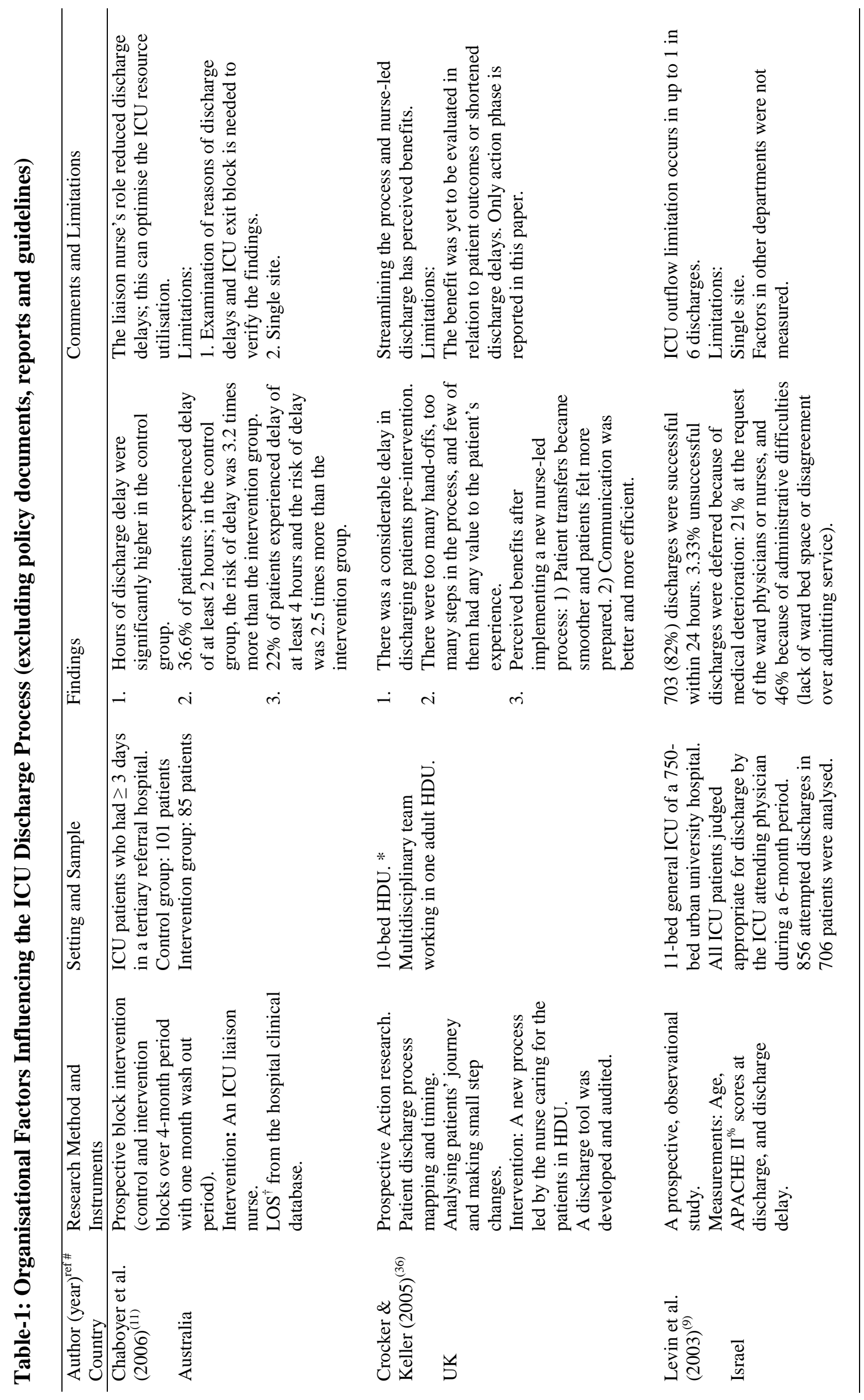




\section{Individual factors}

Table 2 outlines five articles found on individual factors influencing the ICU patient discharge process. Heidegger et al. ${ }^{[27]}$ reported that lack of agreement in clinical decision making exists around ICU patient discharge. Brand ${ }^{[39]}$ found that critical care nurses played a very important and proactive role in bed management, especially when there was pressure on bed availability when discharging patients from the HDU. However, they were not comfortable in contributing to the patient discharge decision-making process, as they saw it as a medical responsibility. Watts et al. ${ }^{[40]}$ found that $9 \%$ of critical care nurses claimed lack of knowledge was one of the important factors impeding the discharge planning process in critical care, consistent with earlier studies. [41] Other studies found that the main reason that ICU patients could not be discharged was the ward staff's lack of knowledge and skills to look after the higher acuity patient. ${ }^{[9,11,30,42,43]}$ Chaboyer et al. ${ }^{[44]}$ found that an ICU liaison nurse helped ward nurses to feel more equipped with knowledge and skills, and more confident about accepting patients from ICU. 


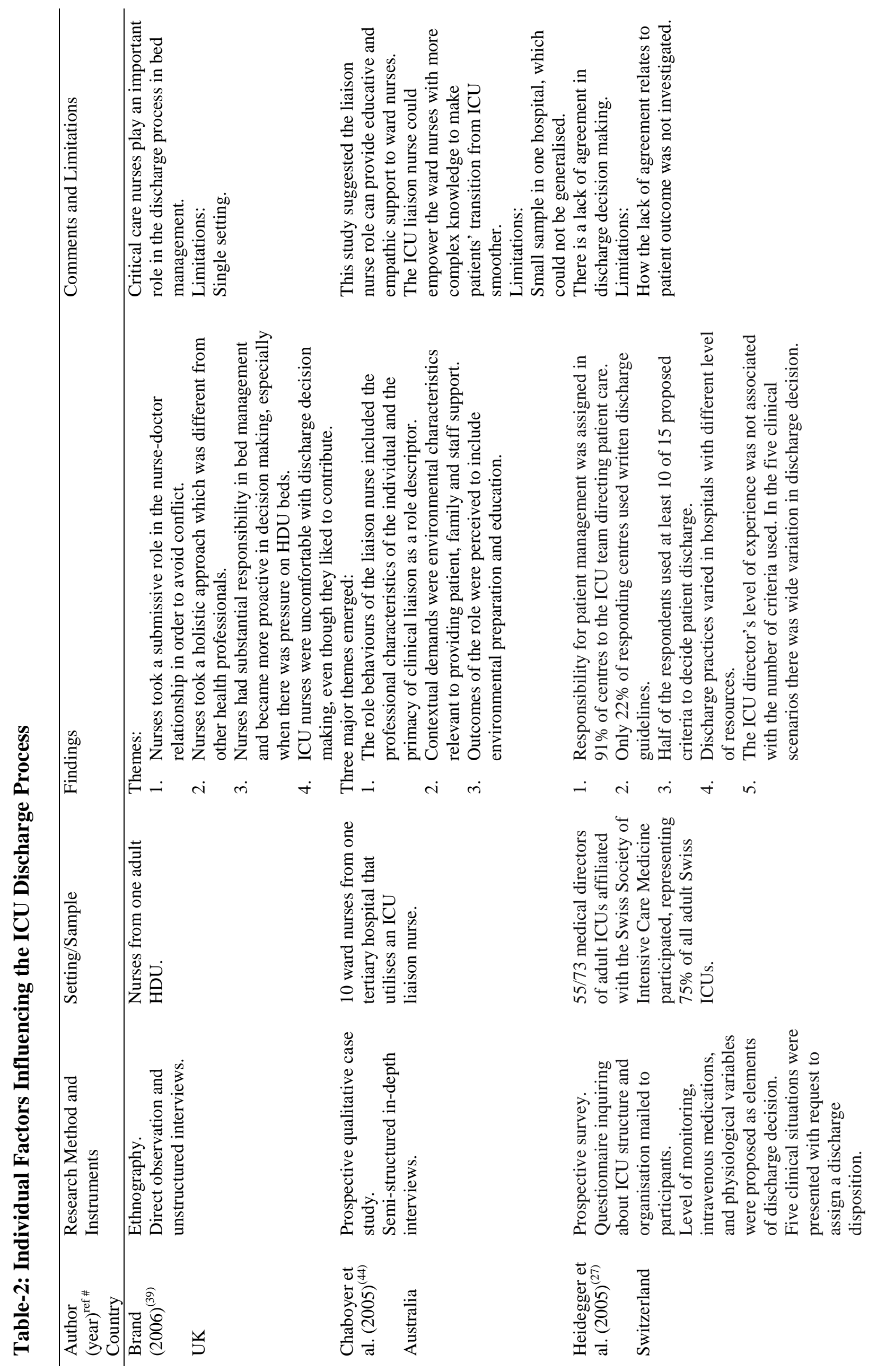




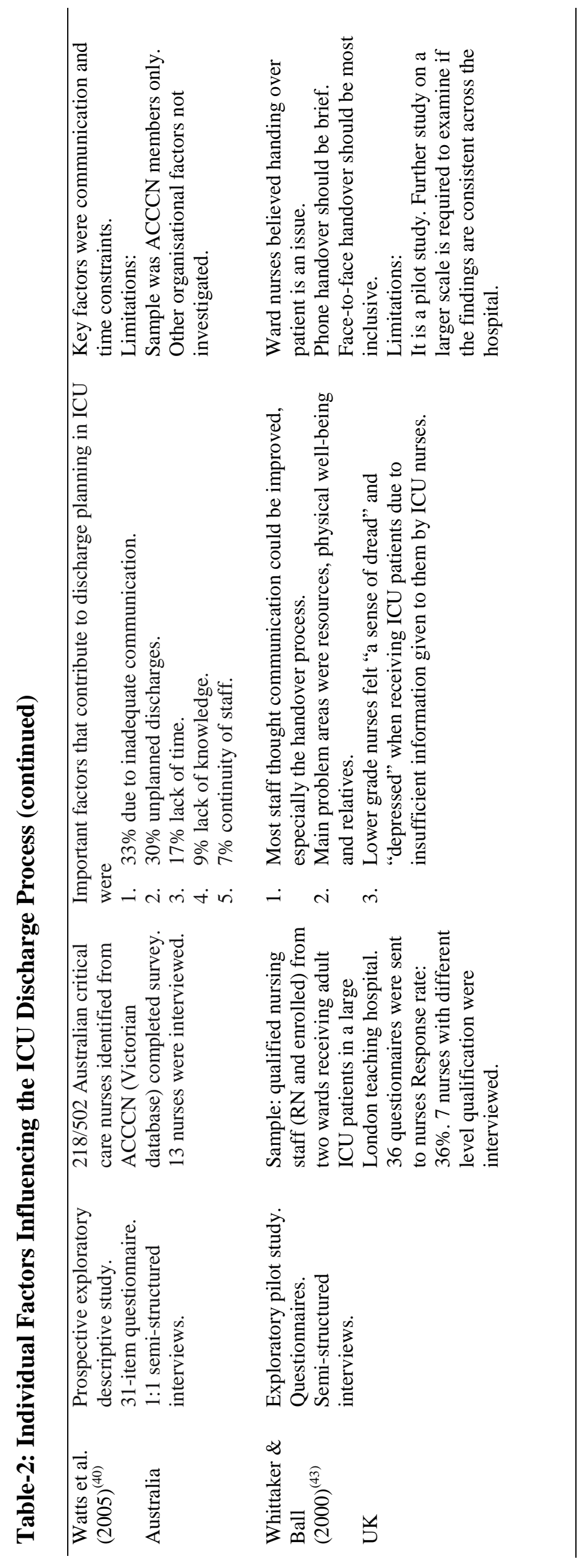




\section{Teamwork}

Table 3 listed six articles regarding teamwork factors related to ICU processes. The role of effective teamwork in accomplishing complex tasks has been well studied. ${ }^{[45]}$ In large organisations, teams make fewer mistakes when each team member understands their own roles and responsibilities. ${ }^{[46-48]}$ By using a daily goals form for ICU patient care, Pronovost ${ }^{[49]}$ found that when team members understood the goals better, ICU length of stay decreased from 2.2 days to 1.1 days. These findings were supported by Jain et al. ${ }^{[50]}$ who found that a multidisciplinary team involvement in daily goal setting for ICU patient care, bed management, and best practice promotion reduced adverse events, and further reduced the cost and length of stay.

In a study examining causes of human errors in ICU, Donchin et al. ${ }^{[51]}$ suggested that the problems of communication between physicians and nurses could contribute to many dangerous human errors. The ICUs that encouraged open communication among team members and across teams were found to perform better in terms of patient length of stay. ${ }^{[52]} \mathrm{Nap}^{[53]}$ found that improved communication and collaboration among ICU doctors and nurses through team training significantly decreased patient ICU mortality. Lack of communication ${ }^{[40,43]}$ or too many unnecessary steps in handing off patients ${ }^{[36]}$ in ICU/HDU patient discharge were perceived as barriers to efficient discharge. However, others found that improved collaboration through improved teamwork was either not associated with outcome ${ }^{[54]}$ or the associations were not measured. $^{[55,56]}$ 


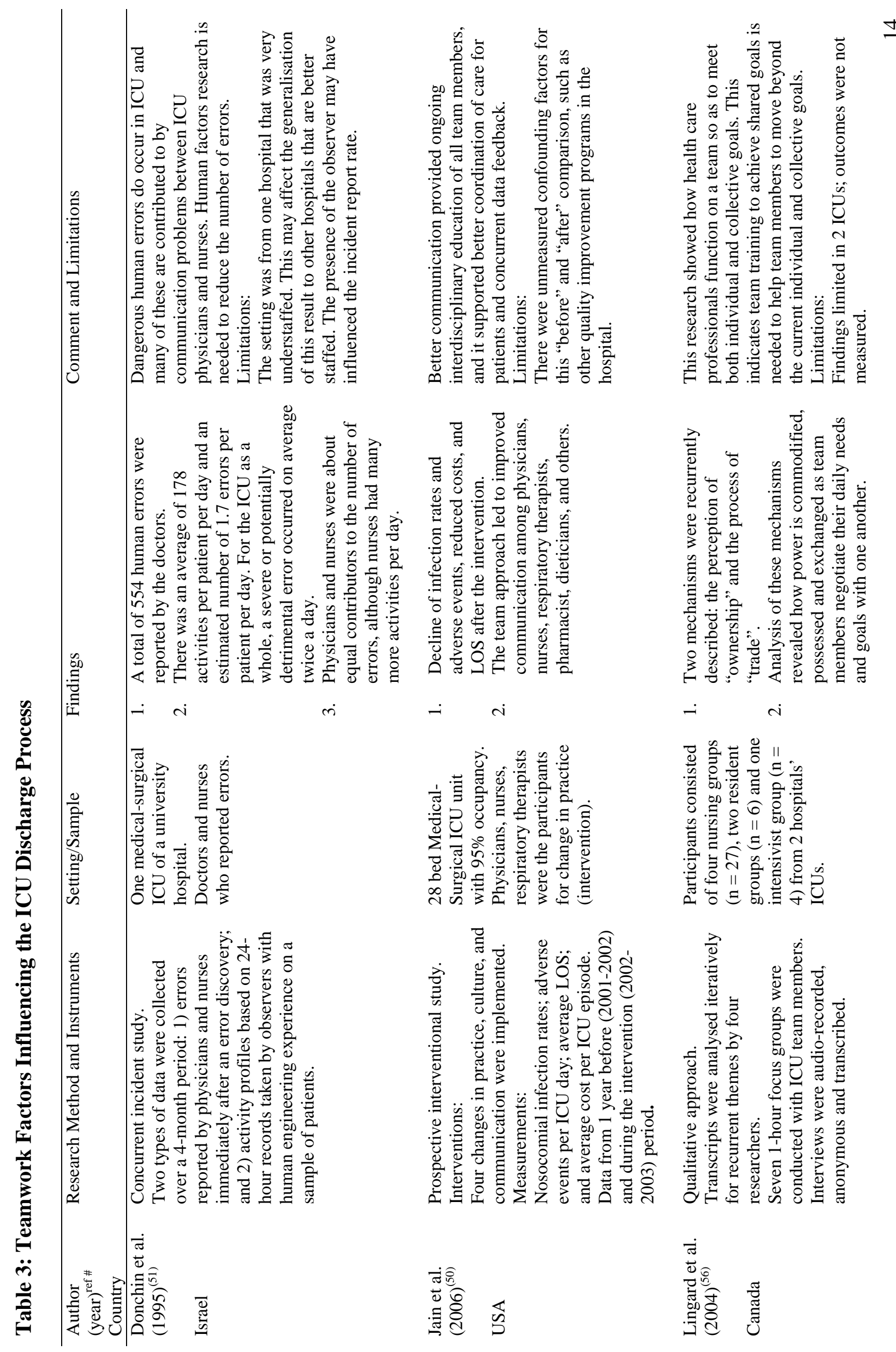




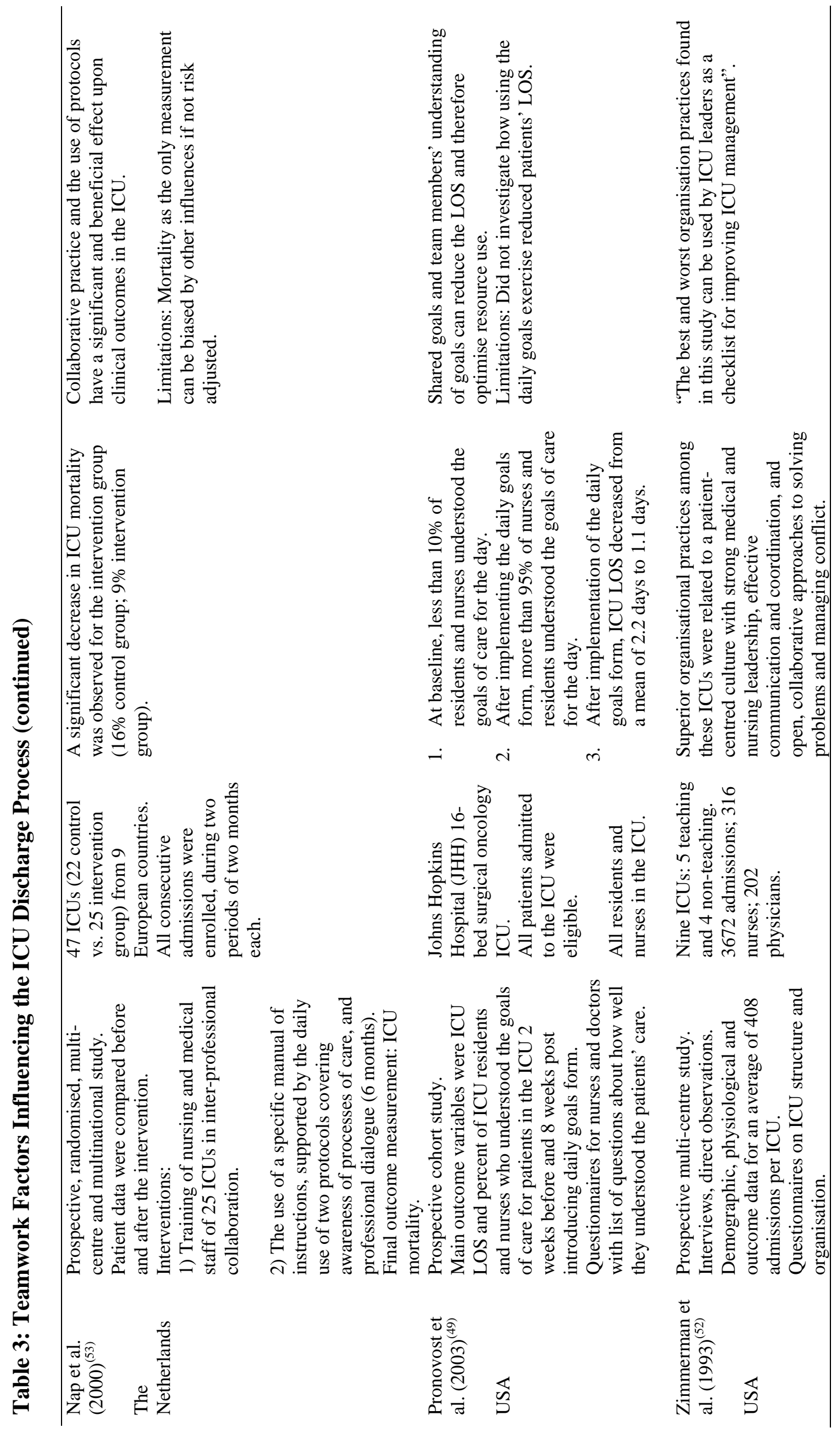




\section{DISCUSSION}

The ICU patient discharge process often starts from ICU admission when the planning of care is initiated, and does not conclude until the patients have been transferred out to wards, and the responsibility, accountability, and management of the patient has been completely handed over to the ward staff. This process can involve health professionals from many disciplines, including ICU specialist physicians and nurses, ward physicians and nurses, managers from different departments, ward clerks, and support systems such as pathology, radiology, etc. Many miniprocesses are embedded within the ICU discharge process, such as the patient discharge decisionmaking process, preparation of patients for discharge from ICU, and handover processes.

Due to the complexity of the ICU discharge process, problems can occur at any stage. Discharge delay can result if no ward beds are available, no hospital support team is available to help with patient transfer, or the ICU nurse cannot get the patient ready due to staffing issues. Premature discharge may result from decision makers’ poor knowledge and/or experience or pressure for beds from other departments. The patient's condition may deteriorate on the wards when the level of nursing care is less than required. Post-ICU discharge mortality can result from night time discharge ${ }^{[28]}$ premature discharge,${ }^{[30]}$ or from human errors.

Triage related to ICU discharge is used when the demand for ICU resources exceeds the supply. Prioritising and triaging methods can be applied by deciding which patient will benefit more from ICU services. The triage discharge model must be used with caution; while it can be a strategy to free up badly needed ICU beds, ${ }^{[57]}$ it can also lead to premature discharge, which has been found to be associated with increased mortality. ${ }^{[8]}$ 
Some researchers have found an association between higher illness severity scores (e.g. APACHE II) and increased mortality in ICU discharged patients. ${ }^{[18,19]}$ Some may argue that this may relate to the fact that some patients are discharged on palliative care, "Do Not Resuscitate" orders. However, Beck et al. ${ }^{[30]}$ argued that late discharge and high discharge TISS scores ${ }^{[58]}$ are significant indicators of premature discharge. Thus, an evidence-based discharge guideline is needed to safeguard the ICU patient discharge practice.

The ICS ${ }^{[21]}$ and SCCM ${ }^{[20]}$ guidelines share similar admission criteria and focus on patients' clinical needs, and in particular, the need to support organ function, the diagnostic group, objective parameters and stability of physical condition. However, the SCCM guidelines provide much more detailed information regarding discharge than the ICS guidelines. Although the relationship between the use of guidelines and protocols and ICU patient outcome is still unclear, research suggests that the use of discharge guidelines and policies improves the utilisation and availability of ICU resources, and reduces ICU stay. ${ }^{[23-25]}$ Therefore, the use of guidelines and policies such as ICU patient discharge criteria may optimise patient flow and the performance of ICUs. In addition, the lack of agreement related to ICU discharge clinical decision making ${ }^{\text {[27] }}$ indicates a need for more research to clarify ICU discharge criteria.

Resource utilisation and availability were found to play a vital role in ICU patient discharge and outcome. Although abundant research has shown that after-hours discharge $\mathrm{e}^{[8,28-31]}$ and premature discharge $^{[8]}$ are associated with increased mortality, these practices still exist. Discharging sicker patients after hours may indicate the pressure on ICU beds, and may be unavoidable due to resource constraints. However, systems and strategies, such as the outreach team, ${ }^{[34,37,38]}$ need to be put into place to make after-hours discharge safer. This further calls for the need to improve the flow and performance of ICUs, to optimise the utilisation of existing resources. 
The availability of resources not only affects the number of patients a unit can admit, it may also influence the discharge decision-making process in ICU. Sprung et al. ${ }^{[59]}$ suggest that better management of ICU beds and more hospital beds can improve the decision-making process by helping the decision makers to focus on the patient's clinical condition rather than on the availability of hospital resources, further preventing ICU patients from being prematurely discharged. Understandably, ICU patient outflow can be influenced by a lack of resources in other parts of the hospital, including a lack of single rooms in the wards, lack of transport for the patient to go to another facility, or a lack of ward-based clinical decision-making due to a lack of medical cover on the wards, ward nurses skill mix and/or ward staffing levels. ${ }^{\text {[30, 60-62] }}$

Efforts in analysing the ICU processes and improving performance have been shown to make a difference in patient outcome and/or organisational outcome. ${ }^{[11,34,35]}$ Organisational interventions related to ICU patient discharge, such as ICU liaison nurses ${ }^{[11]}$ and ICU outreach teams, ${ }^{[34,37,38]}$ appear to improve the flow and performance, and result in shortened hospital stay and decreased patient hospital mortality. However, the degree to which these services are available is not clear. These services may benefit more patients if integrated into the standard ICU structure.

Early discharge planning may help to improve the resource utilisation by more smoothly discharging patients home. Assessment of a patient's discharge needs in ICU will allow sufficient time to get problems sorted and equipment organised for the patient's timely discharge from hospital. However, research shows that many ICU nurses either do not think discharge planning happens in $\mathrm{ICU}^{[63]}$ or lack knowledge about discharge planning. ${ }^{[41]}$

Research indicates that individual factors related to the multidisciplinary team can play a vital role in many stages of the ICU discharge process. Serious adverse events often involve an individual error together with a few system failures, such as work environment, organisational 
levels, and teamwork. ${ }^{[15]}$ The lack of agreement in discharge decision making may indicate a problem in staff training, role clarification, or the use of discharge guidelines. At the same time, discharge planning in ICU needs to be promoted in clinical practice to ensure a faster and smoother hospital journey for patients. Nursing staff in ICU may need to be trained in patient discharge planning. Patients may be discharged sooner from hospital if discharge planning is initiated earlier. This, in turn, could free up the ward beds that often cause bed block.

Teamwork involves shared organisational goals and coordination of effort among team members and across teams. Team members must work in a coordinated manner to realise their shared goals. Communication within and across teams is often at the centre of ICU and hospital activity. Handover from ICU to ward is an important part of communication among team members to ensure continuity of safe patient care. Although handover is currently close to the top of the patient safety agenda ${ }^{[64]}$ and has been researched, to some extent, within the general hospital setting, ${ }^{[65-67]}$ limited research was found regarding the ICU medical and/or nursing handover undertaken when patients are discharged from ICU.

A team cannot realise its shared goals if the team members do not have teamwork skills. Baker et al. ${ }^{[45]}$ found that teamwork training improved the communication and collaboration in teams. However, healthcare workers are rarely trained to work as teams in current education systems, ${ }^{[45]}$ and empirical evidence on teamwork and team training in ICU patient discharge is rare. Additionally, while there has been increasing interest in individual factors and teamwork in healthcare research, few studies explore the multidisciplinary team's influence on the ICU patient discharge process, team training, collaboration and cooperation.

There are some limitations in the review. It excluded a well researched and reviewed aspect: patient factors that contribute to ICU patient discharge. These were excluded because of the 
complexity of this aspect, and the aim of this review was to focus on areas that maybe amenable to change but are not as frequently considered.

\section{RECOMMENDATIONS AND CONCLUSION}

Intensive care patient discharge is influenced by organisational factors, individual factors and teamwork factors. Organisational interventions are effective in reducing ICU discharge delay and shortening patient hospital stay. However, from the current literature, gaps exist. In order to provide evidence for best clinical practice in critical care, more rigorous research is needed to discover how organisational factors, such as discharge guidelines and policies, individual factors, such as clinical decision making, and teamwork factors, such as patient handover, influence the ICU patient discharge process. 


\section{REFERENCES}

1. Kohn LT, Corrigan JM, Donaldson MS. To err is human: Building a safer health system. Washington, DC: National Academy Press. 2000, 287p.

2. Committee on Quality of Health Care in America. Crossing the quality chasm: A new health system for the 21st century. Washington, DC: National Academy Press. 2001, 337p.

3. Martin J, Anderson T, Turton C, Hart GK, Hicks P. Intensive care resources \& activity: Australia and New Zealand 2003-2005. (2006).

4. $\quad$ Rechner IJ, Lipman J. The cost of caring for patients in a tertiary referral Australian Intensive Care Unit. Anaesth Intensive Care. 2005; 33(4): 477-482.

5. Higlett T, Bishop N, Hart GK, Hicks P. Review of intensive care resources \& activity 2002-2003. (2005).

6. Wild C, Narath M. Evaluating and planning ICUs: methods and approaches to differentiate between need and demand. Health Policy. 2005; 71(3): 289-301.

7. Cook R, Rasmussen J. 'Going solid': a model of system dynamics and consequences for patient safety. Quality and Safety in Health Care. 2005; 14: 130-134.

8. Duke GJ, Green JV, Briedis JH. Night-shift discharge from intensive care unit increases the mortality-risk of ICU survivors. Anaesth Intensive Care. 2004; 32(5): 697-701.

9. Levin PD, Worner TM, Sviri S, Goodman SV, Weiss YG, Einav S, Weissman C, Sprung CL. Intensive care outflow limitation: frequency, etiology, and impact. J Crit Care. 2003; 18(4): 206-211.

10. Levin PD, Sprung CL. The process of intensive care triage. Intensive Care Med. 2001; (27): 1441-1445.

11. Chaboyer W, Thalib L, Foster M, Elliott D, Endacott R, Richards B. The impact of an ICU liaison nurse on discharge delay in patients after prolonged ICU stay. Anaesth Intensive Care. 2006; 34(1): 55-60.

12. Reason J. Education and debate: human error: models and management. $\mathrm{Br} \mathrm{Med} J$. 2000; 320(7237): 768.

13. Reason J. Understanding adverse events: human factors, in Clinical Risk Management, Vincent C, Editor. British Medical Journal Publications: London. 1995, p. 573.

14. Vincent C, Taylor-Adams S, Stanhope N. Framework for analysing risk and safety in clinical medicine. Br Med J. 1998; 316(7138): 1154-1157.

15. Pronovost P, Wu AW, Dorman T, Morlock L. Building safety into ICU care. Journal of Critical Care. 2002; 17(2): 78-85.

16. Reason J. Human error. New York: Cambridge University Press. 1990p.

17. Agency for Healthcare Research and Quality. Glossary. [cited 2007 November 2]; 2007.Available from: http://www.ahrq.gov/.

18. Moreno R, Miranda DR, Matos R, Fevereiro T. Mortality after discharge from intensive care: the impact of organ system failure and nursing workload use at discharge. Intensive Care Med. 2001; 27: 999-1004.

19. Smith L, Orts CM, O'Neil I, Batchelor AM, Gascoigne AD, Baudouin SV. TISS and mortality after discharge from intensive care. Intensive Care Med. 1999; 25: 1061-1065.

20. SCCM. Guidelines for ICU admission, discharge, and triage. Crit Care Med. 1999; 27(3): 633-638.

21. ICS.Standards for intensive care units. (1997). www.dh.gov.uk

22. ANZCA. Minimum standards for intensive care units. (2003). http://www.anzca.edu.au/jficm/resources

23. Holcomb BM, Wheeler AP. New ways to reduce unnecessary variation and improve outcomes in the intensive care unit. Crit Care Med. 2001; 7: 304-311. 
24. Imhoff M. Rationing in intensive care medicine, in Rationing in medicine, Breyer F, Kliemt H,Thiel F, Editors. Springer: Berlin. 2002, p. 135.

25. Kern H, Kox WJ. Impact of standard procedures and clinical standards on costeffectiveness and intensive care unit performance in adult patients after cardiac surgery. Intensive Care Med. 1999; 25: 1367-1373.

26. Pronovost P, Jenckes M, Dorman T, Garrett E, Breslow MJ, Rosenfeld BA, Lipsett PA, Bass EB. Organizational characteristics of Intensive Care Units related to outcomes of abdominal aortic surgery. Journal of American Medical Association. 1999; 281(14): 1310-1317.

27. Heidegger CP, Treggiari MM, Romand JA. A nationwide survey of intensive care unit discharge practices. Intensive Care Med. 2005; 31: 1676-1682.

28. Goldfrad C, Rowan K. Consequences of discharges from intensive care at night. The Lancet. 2000; 355: 1138-1142.

29. Priestap FA, Martin CM. Impact of intensive care unit discharge time on patient outcome. Crit Care Med. 2006; 34(12).

30. Beck DH, McQuillan P, Smith GB. Waiting for the break of dawn? The effects of discharge time, discharge TISS scores and discharge facility on hospital mortality after intensive care. Intensive Care Med. 2002; 28: 1287-1293.

31. Tobin AE, Santamaria J. After-hours discharges from intensive care are associated with increased mortality. Medical Journal of Australia [NLM - MEDLINE]. 2006; 184(7): 334337.

32. Knaus WA, Draper EA, Wagner DP, Zimmerman JE. APACHE II: A severity of disease classification system. Crit Care Med. 1985; 13(10): 818-829.

33. Williams T, Leslie G. Delayed discharges from an adult intensive care unit. Aust Health Rev. 2004; 28(1): 87-97.

34. Ball C, Kirkby M, Williams S. Effect of the critical care outreach team on patient survival to discharge from hospital and readmission to critical care: non-randomised population based study. Br Med J. 2003; 327: 1014-1017.

35. Perlmutter DF, Suico C, Krauss AN, Auld P. Case study. A program to reduce discharge delays in a neonatal intensive care unit. Am J Manag Care. 1998; 4(4): 548-552.

36. Crocker C, Keller R. Nurse-led discharge to the ward from high dependency: a service improvement project. Intensive Crit Care Nurs. 2005; 21: 363-366.

37. Priestley G, Watson W, Rashidian A, Mozley C, Russell D, Wilson J, Cope J, Hart D, Kay D, Cowley K, Pateraki J. Introducing Critical Care Outreach: a ward-randomised trial of phased introduction in a general hospital. Intensive Care Med. 2004; 30: 1398-1404.

38. Pittard AJ. Out of our reach? Assessing the impact of introducing a critical care outreach service Anaesthesiology. 2003; 58: 882-885.

39. Brand SL. Nurses' role in discharge decision making in an high dependency unit. Intensive Crit Care Nurs. 2006; 22: 106-114.

40. Watts R, Gardner H, Pierson J. Factors that enhance or impede critical care nurses' discharge planning practices. Intensive Crit Care Nurs. 2005; 21: 302-313.

41. Chaboyer W, Foster M, Kendall E, James H. ICU nurses’ perceptions of discharge planning: a preliminary study. Intensive Crit Care Nurs. 2002; 18: 90-95.

42. Clarke T, Abbenbroek A, Hardy L. The impact of a high dependency unit continuing education program on nursing practice and patient outcomes. Aust Crit Care. 1996; 9(4): 138-149.

43. Whittaker J, Ball C. Discharge from intensive care: a view from the ward. Intensive Crit Care Nurs. 2000; 16(3): 135-143.

44. Chaboyer W, Gillespie B, Foster M, Kendall M. The impact of an ICU liaison nurse: a case study of ward nurses’ perceptions. J Clin Nurs. 2005; 14: 766-775. 
45. Baker DP, Gustafson S, Beaubien JM, Salas E, Barach P.Medical team training programs in health care. Advances in Patient Safety: From Research to Implementation 4, (2005). http://www.ahrq.gov/qual/advances/ 253-267

46. Salas E, Cannon-Bowers JA. The science of training: a decade of progress. Annu Rev Psychol. 2000; 52: 471-499.

47. Sims DE, Salas E, Burke SC. Is there a "big five" in teamwork? in 19th annual meeting of the Society for Industrial and Organisational Psychology. Chicago. 2004.

48. Smith-Jentsch KA, Salas E, Baker DP. Training team performance-related assertiveness. Personnel Psychology. 1996; 49: 909-936.

49. Pronovost P, Berenholtz S, Dorman T, Lipsett PA, Simmonds T, Haraden C. Improving communication in the ICU using daily goals. J Crit Care. 2003; 18(2): 71-75.

50. Jain M, Miller L, Belt D, King D, Berwick DM. Decline in ICU adverse events, nosocomial infections and cost through a quality improvement initiative focusing on teamwork and culture change. Quality \& Safety Health Care. 2006; 15: 235-239.

51. Donchin Y, Gopher D, Olin M, Badihi Y, Biesky M, Sprung C, Pizov R, Cotev SD. A look into the nature and causes of human errors in the intensive care unit. Crit Care Med. 1995; 23(2): 294-300.

52. Zimmerman J, Shortell SM, Rousseau DM, Duffy J, Gillies RR, Devers K, Wagner D, Draper E. Improving intensive care: observations based on organisational case studies in nine intensive care units: a prospective, multicenter study. Crit Care Med. 1993; 21(10): 1443-1451.

53. Nap RE, Silva Alvaro M, Fidler V, Reis Miranda D. Collaborative practice and clinical outcomes in the ICU. Critical Care. 2000; 4(Suppl 1): P221.

54. Shortell SM, Zimmerman J, Rousseau DM, Gillies RR, Wagner D, Draper E. The performance of ICUs: does good management make a difference? Med Care. 1994; 32(5): 508-525.

55. Oandasan I, Baker GR, Barker K, Bosco C, D'Amour D, Jones L, Kimpton S, LemieuxCharles L, Rodriguez LSM, Tepper J, Way D. Teamwork in healthcare: promoting effective teamwork in healthcare in Canada. Policy synthesis and recommendations. (2006). www.chsrf.ca

56. Lingard L, Espin S, Evans C, Hawryluck L. The rules of the game: interprofessional collaboration on the intensive care unit team. Critical Care. 2004; 8(6): R403 - R408.

57. Garland A. Improving ICU: Part 1. Chest. 2005; 127: 2151-2164.

58. Keene AR, Cullen DJ. Therapeutic Intervention Scoring System: update. Crit Care Med. 1983; 11: 1-3.

59. Sprung CL, Geber D, Eidelman LA, Baras M, Pizov R, Nimrod A, Oppenheim A, Epstein L, Cotev S. Evaluation of triage decisions for intensive care admission. Crit Care Med. 1999; 27(6): 1073-1079.

60. Metcalfe MA, Sloggett A, McPherson K. Mortality among appropriately referred patients refused admission to intensive care units. The Lancet. 1997; 350: 7-12.

61. Williams G, Clarke T. A consensus driven method to measure the required number of intensive care nurses in Australia. Aust Crit Care. 2001; 14: 106-115.

62. Zimmerman J, Wagner D, Draper E, Knaus W. Improving intensive care unit discharge decisions: supplementing physician judgment with predictions of next day risk for life support. Crit Care Med. 1994; 22: 1373-1384.

63. Watts R, Pierson J, Gardner H. How do critical care nurses define the discharge planning process? Intensive Crit Care Nurs. 2005; 21: 39-46.

64. World Health Organisation. Communication during patient hand-overs. Patient Safety Solutions. 2007; 1(3).

65. Bomba DT, Prakash R. A description of handover processes in an Australian public hospital. Aust Health Rev. 2005; 29(1): 68-79. 
66. McFetridge B, Gillespie M, Goode D, Melby V. An exploration of the handover process of critically ill patients between nursing staff from the emergency department and the intensive care unit. Nurs Crit Care. 2007; 12(6): 261-269.

67. Fassett MJ, Hannan TJ, Robertson IK, Bollipo SJ, Fassett RG. A national survey of medical morning handover report in Australian hospitals. Med J Aust. 2007; 187(3): 164165 